\title{
Pristimerin, a Naturally Occurring Triterpenoid, Exerts Potent Anticancer Effect in Colon Cancer Cells
}

\author{
Hee Won Seo ${ }^{\S}$, Ju-Hyung Park ${ }^{\S}$, Ji Yeon Lee, Hyun-Ju Park and Jin-Kyung Kim ${ }^{\dagger}$ \\ Department of Biomedical Science, Catholic University of Daegu, Gyeongsan-Si 38430, Korea
}

\begin{abstract}
Pristimerin is a triterpene compound isolated from plant extracts that reportedly possesses antitumor, antioxidant, and anti-inflammatory activities. The current study was designed to evaluate the antitumor effects of pristimerin on human colon cancer cells. Treatment of the human colon cancer cells, HCT116 and SW480, with pristimerin led to a dosedependent decrease in cell proliferation. Flow cytometry experiments showed that pristimerin increased cell apoptotic rate and decreased the mitochondrial membrane potential in HCT116 and SW480 cells. Western blot assay showed that pristimerin induced increased cleavage of caspase-3, $-7,-8$, and poly ADP ribose polymerase. Treatment with pristimerin also caused a marked decrease in the expression of Bcl-2 and Bcl-xL. Additionally, the levels of phosphorylated AKT and forkhead box O3a (FOXO3a) were decreased in pristimerin-treated colon cancer cells. Taken together, our study illustrated that pristimerin promoted apoptosis via the AKT/FOXO3a signaling pathway in colon cancer cells, elucidating that it might be considered as a potential agent for colon cancer therapy.
\end{abstract}

Key Words: Apoptosis, AKT, Colon cancer cells, Forkhead box O3a, Pristimerin

\section{INTRODUCTION}

In 2015, there were 1.7 million incident cases of colon and rectum cancer, and it caused 832,000 deaths. Colorectal cancer caused 17 million and disability-adjusted life-years in 2015 of which $96 \%$ came from years of life lost and 4\% came from years lived with disability (Nagai and Kim, 2017). The odds of developing colorectal cancer before age 79 years at the global level was higher for men than for women. Globally colorectal cancer ranked third for cancer incidence and second for cancer deaths in 2015 (Nagai and Kim, 2017). Although there are many ways for management colorectal cancer such as screening, surgical management, irradiation and adjuvant chemotherapy, it remains one of the most lifethreatening malignancies, particularly when it becomes in advanced stages (Brenner et al., 2014). Thus, discovering of effective and safe compounds urgently needed to reduce colorectal cancer mortality.

During the course of chemopreventive agent identification, phytochemicals draw extensive attention for their relative safety (Lee et al., 2011). Pristimerin is a quininemethide triterpenoid compound that has been found in various species belonging to Celastraceae and Hippocrateaceae families. It displays a variety of biological activities, including antifungal, antioxidant, antibacterial, and anti-inflammatory properties (Carvalho et al., 2005; López et al., 2011; Gullo et al., 2012; Kim et al., 2013). It also has been reported that pristimerin,

* Received: January 26, 2018 / Revised: February 14, 2018 / Accepted: February 23, 2018

$\S$ These authors contributed equally to this work.

${ }^{\dagger}$ Corresponding author: Jin-Kyung Kim. Department of Biomedical Science, Catholic University of Daegu, 13-13 Hayang-Ro, Gyeongsan-Si 38430, Korea. Tel: +82-53-850-3774, Fax:+82-53-850-3774, e-mail: toto0818@cu.ac.kr

(C) The Korean Society for Biomedical Laboratory Sciences. All rights reserved.

(C) This is an Open Access article distributed under the terms of the Creative Commons Attribution Non-Commercial License (http://creativecommons.org/licenses/by-nc/3.0/) which permits unrestricted non-commercial use, distribution, and reproduction in any medium, provided the original work is properly cited. 
as a new proteasome inhibitor, has promising clinical potential as both a therapeutic and chemopreventive agent for cancer (Tiedemann et al., 2009). Indeed, pristimerin inhibited tumor growth of various human cancers such as breast, prostate, pancreatic, hepatic and multiple myeloma tumors (Yousef et al., 2017). In addition, a recent study reported that pristimerin inhibited cell cycle progression and induced apoptosis in colon cancer cells though blocking EGFR and HER2 activation (Yousef et al., 2016). In the present study, we further investigated anticancer potential of pristimerin on human colon cancer cells, HCT116 (as p53 normal cells) and SW480 (as p53 mutant cells) cells. In addition, the molecular mechanism of anticancer effect of pristimerin was demonstrated in both colon cancer cells.

\section{MATERIALS AND METHODS}

\section{Reagents}

Pristimerin was purchased from Santa Cruz Biotechnology (Santa Cruz, CA, USA). RPMI-1640 medium, fetal bovine serum (FBS), penicillin, streptomycin, and trypsin/EDTA were purchased from Hyclone (Logan, UT, USA). Dimethylsulfoxide (DMSO) and anti- $\beta$-actin antibody were purchased from Sigma Aldrich (St. Louis, MO, USA). Protein detection BCA kit was obtained from Thermo Scientific Pierce (Rockford, IL, USA). For Western blot analysis, following antibodies were used: anti-cleaved caspase-3 rabbit polyclonal, anti-cleaved caspase-7 rabbit polyclonal, anti-cleaved caspase- 8 rabbit monoclonal, anti-cleaved caspase- 9 rabbit polyclonal, anti-cleaved-PARP rabbit polyclonal, anti-BAX rabbit polyclonal, anti-tBid rabbit polyclonal, anti-phosphoAKT (p-AKT) rabbit monoclonal, anti-phospho-forkhead box O3a (p-FOXO3a) rabbit polyclonal, anti-FOXO3a antibody rabbit monoclonal (all Cell Signaling Technology, Danvers, USA), anti-Bcl-2 rabbit polyclonal, anti-Bcl-xL rabbit polyclonal (all Santa Cruz Biotechnology, Santa Cruz, USA).

\section{Cells and cell culture}

Human HCT116 and SW480 colon cancer cells were obtained from the Korean Cell Line Bank (Seoul, Korea). The cells were maintained in RPMI 1640 medium at $37^{\circ} \mathrm{C}$ with $5 \% \mathrm{CO}_{2}$ in a humidified atmosphere. All media were supplemented with $10 \%$ fetal bovine serum, $100 \mathrm{U} / \mathrm{mL}$ penicillin and $100 \mu \mathrm{g} / \mathrm{mL}$ streptomycin. The cells attained 70\% confluence with a normal morphology and multiplication pattern, and were used for experiments between passages 3-12. Unless otherwise mentioned, control treatment refers to the cells treated with an equal amount of DMSO; the maximum amount of DMSO was maintained at $<0.1 \% \mathrm{v} / \mathrm{v}$.

\section{Cell proliferation assay}

HCT116 and SW480 cells were seeded into wells of 96-well plates at a density of $1 \times 10^{4}$ cells/well. The rate of cell proliferation was determined at $24 \mathrm{~h}$ with CellTiter $96^{\circledR}$ AQueous One Solution Cell Proliferation assay (Promega, Madison, WI, USA) according to the manufacturer's instruction. Absorbance at $490 \mathrm{~nm}$ was measured with a microplate reader, and the average absorbance values were calculated.

\section{Annexin V apoptosis assay}

Cells $\left(1 \times 10^{5}\right.$ per well $)$ in 24 -well plates treated with indicated concentration of pristimerin were harvested, pooled in complete medium and washed repeatedly with sterile phosphate buffered saline (PBS). Cells suspended in PBS and stained with Muse ${ }^{\circledR}$ Annexin V and Dead Cell Assay Kit (Merck Millipore, Billerica, MA, USA) according to the manufacturer's instruction. The stained cells were analyzed immediately by using Muse ${ }^{\circledR}$ Cell Analyzer (Merck Millipore).

\section{Measurement of mitochondrial membrane potential (MMP)}

MMP was determined using a JC-1 mitochondrial membrane potential detection kit (Biotium, Hayward, CA, USA) according to the manufacturer's instructions. Briefly, cells $\left(1 \times 10^{5}\right.$ per well $)$ were plated in 24 -well plates. After $24 \mathrm{~h}$ incubation with indicated concentrations of pristimerin, cells were collected and mixed with $0.5 \mathrm{~mL}$ of JC-1 working solution at $37^{\circ} \mathrm{C}$ in the dark for $15 \mathrm{~min}$. Cells were analyzed immediately by flow cytometry (Becton Dickinson, Franklin Lakes, NJ, USA) using FlowJo software (TreeStar Inc., Ashland, OR, USA). 
(A)

B<smiles>CC(=O)[C@]1(C)CC[C@H]2CC[C@]3(C)C4=CC=C5C(=CC(=O)C(O)=C5C)[C@H]4CC[C@]3(C)[C@H]2C1</smiles>

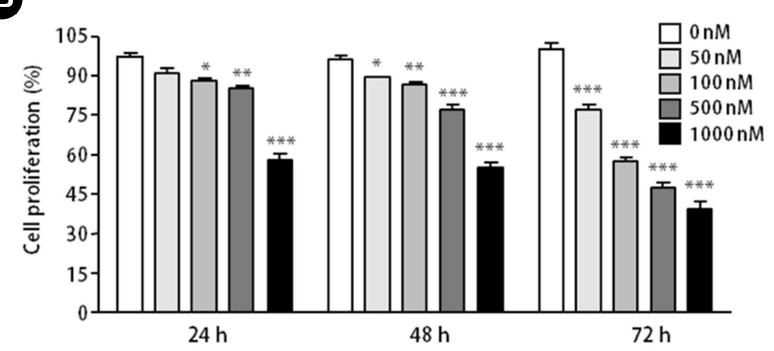

C

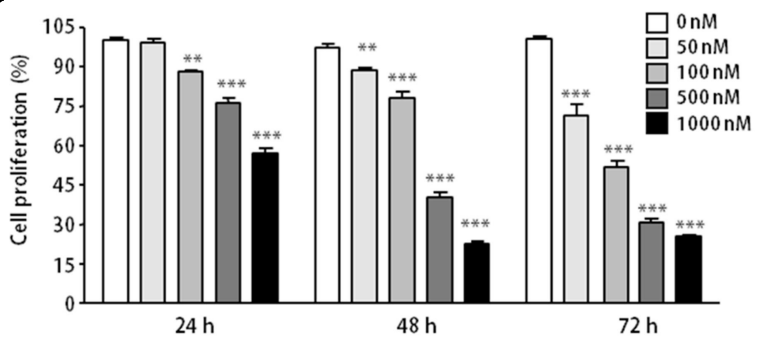

Fig. 1. Effects of pristimerin on colon cancer cell proliferation. (A) The structure of pristimerin. (B) HCT116 cells and (C) SW480 cells were treated with indicated concentration of pristimerin for 24,48 and $72 \mathrm{~h}$, after which the proliferation was determined as described in Methods. The results are reported as the mean \pm S.E.M. of three independent experiments in triplicate. Statistical significance is based on the difference when compared with $0 \mathrm{nM}$ treated cells, ${ }^{*} P<0.05,{ }^{* *} P<0.01,{ }^{* * *} P<0.001$.

\section{Western blot analysis}

Cells were lysed in Pro-prep protein extraction solution (iNtRon, Sungnam, Korea) containing $1 \times$ protease and phosphatase inhibitor cocktail. The protein concentrations were determined using a BCA protein assay kit. Whole cell extracts were separated by $12 \%$ or $15 \%$ sodium dodecyl sulfate-polyacrylamide gel electrophoresis and transferred to a PVDF membrane. After blocking with 3\% skim milk for $1 \mathrm{~h}$, the membranes were incubated with primary antibody at $4{ }^{\circ} \mathrm{C}$ overnight. The membranes were then washed with PBST (1× PBS, $0.1 \%$ Tween 20$)$ and incubated for $1 \mathrm{~h}$ with anti-rabbit IgG or anti-mouse IgG conjugated with HRP. After washing, the protein bands were then visualized using a WEST-One ${ }^{\mathrm{TM}}$ Western Blot Detection Spray (iNtRon) and DAVINCH-Chemi CAS-400SM (Davinch-k, Seoul, Korea). Dates were assessed using Total Lab software (Davinch-k).

\section{Statistical analysis}

Values are expressed as the mean \pm S.E.M. of the results of at least three experiments. The values were then evaluated by one-way analysis of variance (ANOVA) with Bonferroni multiple comparison post tests using the GraphPad Prism 4.0 software (GraphPad Software, San Diego, CA, USA). Differences with $P$ values $<0.05$ were considered statistically significant.

\section{RESULTS}

\section{Pristimerin inhibited cell proliferation in human colon} cancer cells

To evaluate anticancer effect of pristimerin in colon cancer cells, we firstly examined whether pristimerin affected the proliferation of HCT116 and SW480 cells. These cells were treated with different concentrations $(0 \sim 1,000 \mathrm{nM})$ of pristimerin for 24,48 , or $72 \mathrm{~h}$ and cell proliferation was assessed by the MTS assay. As shown in Fig. $1 \mathrm{~B}$ and $1 \mathrm{C}$, pristimerin treatment produced a dose-dependent and time-dependent inhibition of the proliferation of colon cancer cells.

\section{Pristimerin induces apoptosis of human colon cancer cells}

To determine if apoptosis mediated the inhibition of HCT116 and SW480 proliferation, the loss of phosphatidylserine asymmetry was quantified by cytometry of 7-AAD/Annexin V double-stained cells. HCT116 and SW480 cells treated with 500 and 1,000 $\mathrm{nM}$ pristimerin for $24 \mathrm{~h}$ were significantly increased Annexin V-positive cells indicating that pristimerin induced apoptosis (Figs. 2A-2C).

Apoptosis was further examined by measuring cleaved caspases and PARP in HCT116 and SW480 cells treated with pristimerin for $24 \mathrm{~h}$ because activation of caspases and the subsequent cleavage of PARP are classic molecular markers of apoptosis (Elmore, 2007). Consistent with Annexin V staining results, the levels of cleaved caspase-3, $-7,-8$, and -PARP were increased in cells treated with pristimerin (Fig. 
A

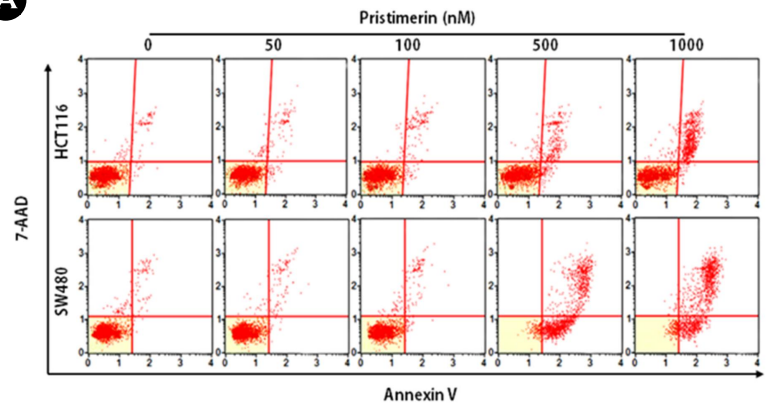

B

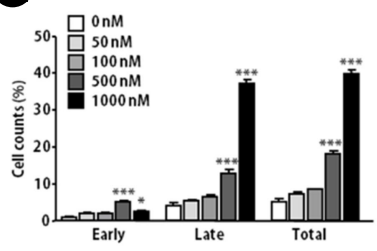

C

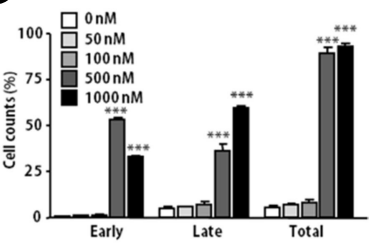

(D)

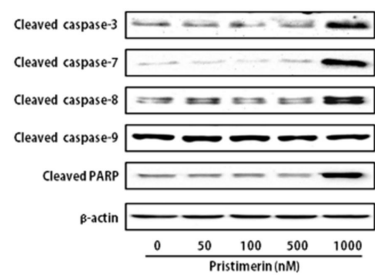

E
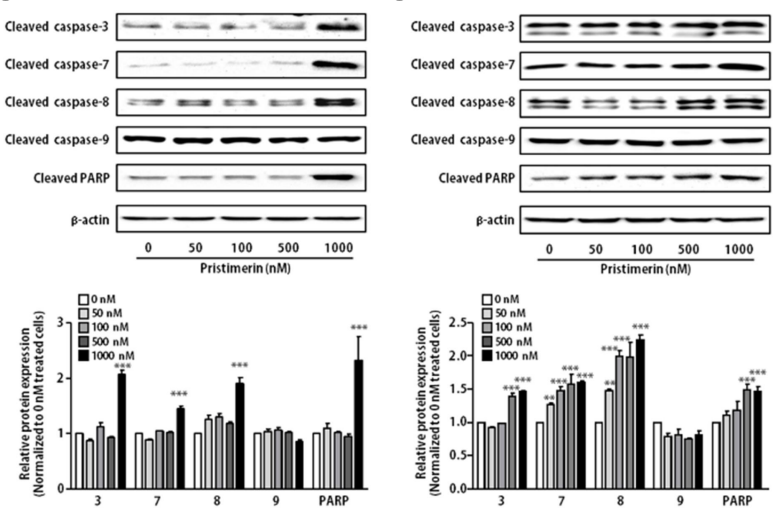

Fig. 2. Pristimerin induces apoptosis in human colon cancer cells. (A) HCT116 and SW480 cells were treated with indicated concentration of pristimerin for $24 \mathrm{~h}$ and apoptosis was evaluated using Annexin V Assay Kit. The lower right quadrant (Annexin $\mathrm{V}^{+} /$ 7-AAD') represents early apoptosis, and the upper right quadrant (Annexin $\mathrm{V}^{+} / \mathrm{PI}^{+}$) represents late apoptotic cells. Percentages of early and late apoptotic cells in (B) HCT116 and (C) SW480 cells are shown. (D) HCT116 and (E) SW480 cells were treated with indicated concentration of pristimerin for $24 \mathrm{~h}$. Equal amounts of cell lysates were subjected to Western blot analysis for indicated proteins. $\beta$-actin was served as an equal loading control. Relative protein expression is presented in the bar graphs below. The results shown are the mean \pm S.E.M. of four independent experiments. Statistical significance is based on the difference when compared with $0 \mathrm{nM}$-treated cells, ${ }^{*} P<0.05$, ${ }^{* *} P<0.01,{ }^{* * *} P<0.001$.

$2 \mathrm{D}$ and $2 \mathrm{E}$ ). In contrast, no detectable changes in amount of cleaved caspase-9 following pristimerin treatment were observed.
Treatment of pristimerin reduces the mitochondrial membrane potential (MMP)

Cell apoptosis appears to be controlled by Bcl-2 family proteins (Elmore, 2007; Kale et al., 2018). Bcl-2 protein is a cell death inhibitor, whereas BAX has a critical role in facilitating apoptosis. Thus, we measured the expression of Bcl-2 family proteins in HCT116 and SW480 cells by Western blot analysis after treatment with pristimerin. The expression of Bcl-2 and Bcl-xL was decreased, while the levels of BAX were unchanged (Fig. 3A and 3B). The expression of tBid was also elevated by pristimerin treatment (Fig. 3A and 3B). These results suggest that the apoptotic effect of pristimerin may be mediated by Bcl-2, Bcl-xL and tBid.

Disruption of the function of Bcl-2 protein leads to permeabilization of MMP. We therefore investigated the effects of pristimerin on MMP using JC-1 fluorescent dye and flow cytometry. JC-1, a cationic dye, produces red fluorescent J-aggregates in mitochondria with high MMP and green fluorescence with low MMP. An increased proportion of HCT116 and SW480 cells experiencing mitochondrial depolarization and undergoing cell death. Exposure of colon cancer cells to 500 and $1,000 \mathrm{nM}$ pristimerin for $24 \mathrm{~h}$ led to a significant reduction in the MMP (Fig. 3C and 3D).

\section{Role of AKT and FOXO3a signaling pathway in pristimerin-treated colon cancer cells}

As reported, AKT is constitutively activated in majority of colon cancer (Manning and Toker, 2017). Therefore, we hypothesized that the anticancer effect of pristimerin in colon cancer cells was due to inhibition of AKT pathway. To test our hypothesis, constitutive protein and phosphorylation levels of AKT were examined in both colon cancer cells by Western blot analysis. As shown in Fig. 4, phosphorylation of AKT was dramatically suppressed by 100,500 and 1,000 nM pristimerin treatment. However, there was no variation in the expression of AKT. Next, we investigated FOXO3a, which is an important downstream molecule of AKT pathway and a tumor suppressor in the human cancers (Link et al., 2017; Manning and Toker, 2017). The results showed that the phosphorylated levels, but not protein levels of FOXO3a 
(A)
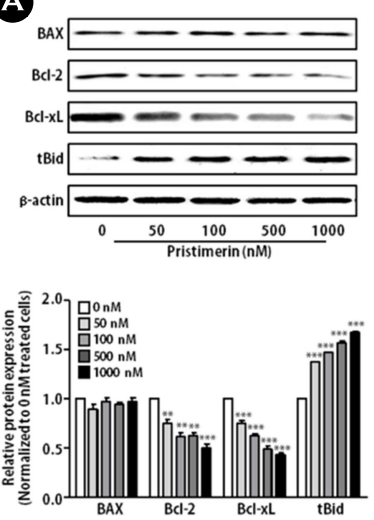

C

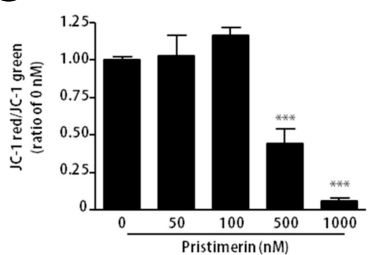

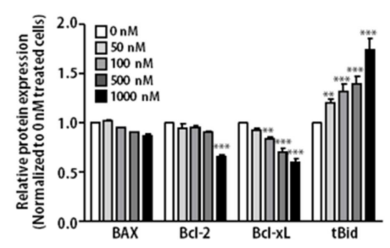

B

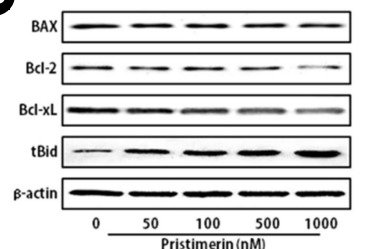

D

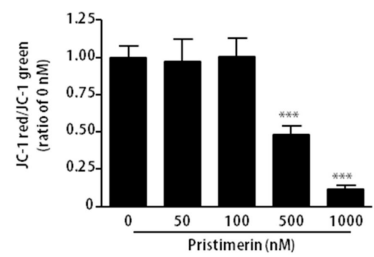

Fig. 3. The dissipation of mitochondrial membrane potential (MMP) of colon cancer cells upon treatment with pristimerin. Western blot analysis showing BAX, Bcl-2, Bcl-xL and tBid protein levels in (A) HCT116 and (B) SW480 cells with the same treatments as in Fig. 2. Equal amounts of cell lysates were were subjected to Western blot analysis for indicated proteins. $\beta$-actin was served as an equal loading control. Relative protein expression is presented in the bar graphs below. HCT116 (C) and SW480 cells (D) treated with indicated concentrations of pristimerin for $24 \mathrm{~h}$ were stained with JC-1 and the average ratio of red/green fluorescence intensity was analyzed by flow cytometry. The data shown are representative of three independent experiments. Relative protein expression is presented in the bar graphs below. The results shown are the mean \pm S.E.M. of four independent experiments. Statistical significance is based on the difference when compared with 0 nM-treated cells, $* * P<0.01, * * * P<0.001$.

were decreased in pristimerin-treated cells (Fig. 4). The results indicated that pristimerin specifically targeted the activation of AKT/FOXO3a pathway.

\section{DISCUSSION}

Colon cancer is the third most common malignancy both in terms of estimated new cases and estimated deaths in 2016 (Siegel et al., 2016). Despite the development of several therapies for its treatment, there is still no effective cure for patients with advanced stages of the disease and the prognosis for advanced tumors remains reserved. Therefore, more

A

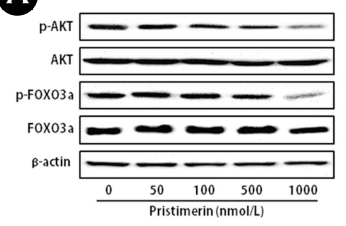

B
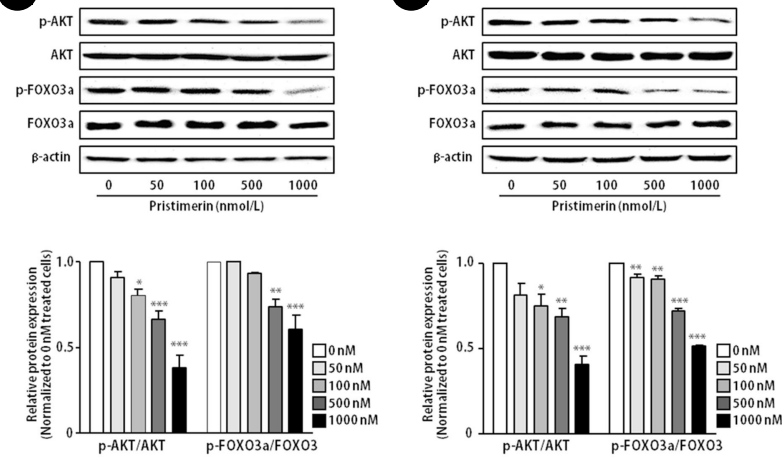

Fig. 4. Effect of pristimerin on the activation of AKT and FOXO3a signaling pathway. (A) HCT and (B) SW480 cells were treated with indicated concentration of pristimerin for $24 \mathrm{~h}$. Equal amounts of cell lysates were subjected to Western blot analyses for p-AKT, AKT, p-FOXO3a and FOXO3a. Bar diagram showed the quantitation of respective Western blots. Each bar represents means \pm S.E.M. of at least three independent experiments. Statistical significance is based on the difference when compared with $0 \mathrm{nM}$-treated cells, $* P<0.05, * * P<0.01, * * * P<0.001$.

studies are still needed to develop non-toxic new antitumor approaches with minimal side effects. Phytochemicals constitute a prolific source of therapeutic compounds that exhibit antitumor activities.

In present study, we found that pristimerin suppressed the proliferation of colon cancer cells in a dose-dependent manner in both HCT116 and SW480 cells (Fig. 1). By flow cytometry using Annexin V binding assay, we demonstrated that pristimerin triggers apoptosis in HCT116 and SW480 cells (Figs. 2A-2C). This effect implies increased expression level of cleaved caspase-3, -7, -8 and -PARP, a hallmark of apoptosis (Fig. 2D and 2E). The extrinsic pathway of apoptosis is initiated by the binding of ligands to death receptors. The transmembrane death signal induces formation of the death-inducing signaling complex through adaptor proteins, subsequently leading to caspase-8 activation (Elmore, 2007). Presently, caspase- 8 also played a role in apoptosis of colon cancer cells treated with pristimerin.

The p53 protein becomes stabilized and activated in response to a number of stimuli, including exposure of cells to DNA-damaging agents and oncogene activation. The activation of p53 allows it to carry out its function as a tumor suppressor through cell cycle arrest, apoptosis, DNA repair, differentiation, and antiangiogenesis (el-Deiry, 1998; Wang 
et al., 2015). Because of these abilities, the tumor suppressor p53 is an attractive target in cancer therapy (Bo et al., 2014). However, most of the tumor malignancies are associated with mutation of p53 gene and the state of p53 is pivotal for the response of tumor cells to anticancer therapies (Muller and Vousden, 2013). Many studies indicated that colon cancer cells with mutant p53 were more chemo-resistant than those with wild type p53 due to their poor ability to activate apoptosis and initiation of apoptosis is the most important functions of p53 (Iacopetta, 2003; Chen, 2016). There is no correlation between cell sensitivity to pristimerin and p53 status, because the anti-proliferative effect of pristimerin for SW480 cells, which carry mutant p53 are similar to those for HCT116 cells, which have wild-type p53 (Figs. 1B and 1C). Interestingly, pristimerin was able to affect the viability of SW480 colon adenocarcinoma cells that express a p53 mutated form $(\mathrm{R} 273 \mathrm{H})$, a very well-known hot spot mutation in tumors. Besides, the percentage of apoptotic cells in pristimerin-treated SW480 cells was much higher than HCT116 cells (Figs. 2A-2C). This result suggests that pristimerin effects on the viability of colon cancer cells did not rely on $\mathrm{p} 53$. Also, pristimerin might be a potential agent to induce apoptosis against p53 mutant cancer cells.

The intrinsic apoptotic pathway involve non receptor mediated stimuli that produce mitochondrial mediated signals, resulting in an opening of mitochondrial permeability transition pore, loss of the MMP and release of pro-apoptotic proteins such as cytochrome c. The Bcl-2 family of proteins, which includes anti-apoptotic proteins such as Bcl-2 and Bcl-xL and pro-apoptotic proteins such as BAX and Bid regulates the release of cytochrome $\mathrm{c}$ from mitochondria via control of the mitochondrial membrane's permeability. Release of cytochrome c contributes to the activation of caspase-9 followed by sequential activation of caspase- 3 and cleavage of PARP (Decker and Muller, 2002; Elmore, 2007). Our results showed that pristimerin induced apoptosis of colon cancer cells was associated with the down regulation of the Bcl-2 level and the up regulations of the tBid levels (Fig. 3A and 3B), as well as the loss of MMP (Fig. 3C and $3 \mathrm{D})$, indicating that pristimerin induced mitochondrial dysfunction through regulation of the Bcl-2 family, leads to apoptosis.
Pristimerin did not change the levels of cleaved caspase-9 in both colon cancer cells (Fig. 2D and Fig. 2E). Until recently, the sole apical initiator in apoptosis pathways was assumed to be caspase-9, which is activated in a complex termed the apoptosome by the scaffold protein Apaf-1 and its cofactor cytochrome c (Shakeri et al., 2017). Caspase-9 is of particular interest because it initiates cell death in response to noncytokine-mediated cellular stress, such as UV or chemical treatment (Sakai et al., 2004). However, the apoptosome is not universally essential for Bcl-2-regulated apoptosis, because certain cells lacking Apaf-1 or caspase-9 readily undergo apoptosis in response to diverse insults (Marsden et al., 2002; Tomicic et al., 2005). Here we found that pristimerinmediated apoptosis in HCT116 and SW480 cells was independent of caspase-9. As processing of this caspase did not occur, it is possible that the other initiator caspase may be involved in pristimerin-induced apoptosis.

FOXO3a, a member of FOXO transcription factors family, is a promising target to evaluate the tumorigenesis in different cancer types and it is required to induce the expression of various genes necessary for cell death (Fernández de Mattos et al., 2008). The major upstream regulator of FOXO3a is AKT signaling axis (Manning and Toker, 2017). AKTdependent phosphorylation of FOXO3a leads to its cytoplasmic retention by 14-3-3 proteins and loss of target gene activation (Manning and Toker, 2017). AKT-mediated phosphorylation of FOXO3a promotes cell survival through inhibiting proapoptotic proteins such as TRAIL, Bim and promoting anti-apoptosis protein such as Bcl-xL (Fernández de Mattos et al., 2008). Our results reveal that the phosphorylation of FOXO3a, but not protein expression, was decreased with pristimerin treatment in colon cancer cells (Fig. 4A and 4B). Previous study also showed decreased levels of phospho-FOXO3a in pristimerin-treated pancreatic cancer cells (Deeb et al., 2014). Furthermore, FOXO3a responsive Bcl-xL were decreased by pristimerin. These results suggest that pristimerin induces apoptosis by inhibiting the AKT/ FOXO3a pathway. This evidence showed that pristimerin was efficacious for the growth control of colon cancer cells, demonstrated the potential utility of pristimerin in clinical settings. 


\section{ACKNOWLEDGEMENTS}

This work was supported by a National Research Foundation of Korea grant funded by the government of Korea (NRF-2017R1A2B1006771).

\section{CONFLICT OF INTEREST}

The authors declare no conflicts of interest.

\section{REFERENCES}

Bo H, van den Heuvel APJ, Varun VP, Shengliang Z, Wafik SED. Targeting tumor suppressor p53 for cancer therapy: Strategies, challenges and opportunities. Current Drug Targets. 2014. 15: 80-89.

Brenner H, Kloor M, Pox CP. Colorectal cancer. Lancet. 2014. 383: $1490-1502$.

Carvalho PR, Silva DH, Bolzani VS, Furlan M. Antioxidant quinonemethide triterpenes from Salacia campestris. Chemistry \& Biodiversity. 2005. 2: 367-372.

Chen J. The cell-cycle arrest and apoptotic functions of p53 in tumor initiation and progression. Cold Spring Harbor Perspectives in Medicine. 2016. 6: a026104.

Decker P, Muller S. Modulating poly (ADP-ribose) polymerase activity: potential for the prevention and therapy of pathogenic situations involving DNA damage and oxidative stress. Current Pharmaceutical Biotechnology. 2002. 3: 275-283.

Deeb D, Gao X, Liu YB, Pindolia K, Gautam SC. Pristimerin, a quinonemethide triterpenoid, induces apoptosis in pancreatic cancer cells through the inhibition of pro-survival Akt/ $\mathrm{NF}-\kappa \mathrm{B} / \mathrm{mTOR}$ signaling proteins and anti-apoptotic Bcl-2. International Journal of Oncology. 2014. 44: 1707-1715.

el-Deiry WS. Regulation of p53 downstream genes. Seminars in Cancer Biology. 1998. 8: 345-57.

Elmore S. Apoptosis: a review of programmed cell death. Toxicologic Pathology. 2007. 35: 495-516.

Fernández de Mattos S, Villalonga P, Clardy J, Lam EW. FOXO3a mediates the cytotoxic effects of cisplatin in colon cancer cells. Molecular Cancer Therapeutics. 2008. 7: 3237-3246.

Gullo FP, Sardi JC, Santos VA, Sangalli-Leite F, Pitangui NS, Rossi SA, de Paula E, Silva AC, Soares LA, Silva JF, Oliveira HC, Furlan M, Silva DH, Bolzani VS, Mendes-Giannini MJ, Fusco-Almeida AM. Antifungal activity of maytenin and pristimerin. Evidence-Based Complementary and Alternative Medicine. 2012. 2012: 340787.
Iacopetta B. TP53 mutation in colorectal cancer. Human Mutation. 2003. 21: 271-276.

Kale J, Osterlund EJ, Andrews DW. BCL-2 family proteins: changing partners in the dance towards death. Cell Death \& Differentiation. 2018. 25: 65-80.

Kim HJ, Park GM, Kim JK. Anti-inflammatory effect of pristimerin on lipopolysaccharide-induced inflammatory responses in murine macrophages. Archives of Pharmacal Research. 2013. 36: 495-500.

Lee KW, Bode AM, Dong Z. Molecular targets of phytochemicals for cancer prevention. Nature Reviews Cancer. 2011. 11: 211 -218 .

Link W, Fernandez-Marcos PJ. FOXO transcription factors at the interface of metabolism and cancer. International Journal of Cancer. 2017. 141: 2379-2391.

López MR, de León L, Moujir L. Antibacterial properties of phenolic triterpenoids against Staphylococcus epidermidis. Planta Medica. 2011. 77: 726-729.

Manning BD, Toker A. AKT/PKB Signaling: Navigating the network. Cell. 2017. 169: 381-405.

Marsden VS1, O'Connor L, O'Reilly LA, Silke J, Metcalf D, Ekert PG, Huang DC, Cecconi F, Kuida K, Tomaselli KJ, Roy S, Nicholson DW, Vaux DL, Bouillet P, Adams JM, Strasser A. Apoptosis initiated by Bcl-2-regulated caspase activation independently of the cytochrome c/Apaf-1/caspase-9 apoptosome Nature. 2002. 419: 634-637.

Muller PAJ, Vousden KH. P53 mutations in cancer. Nature Cell Biology. 2013. 15: 2-8.

Nagai H, Kim YH. Cancer prevention from the perspective of global cancer burden patterns. Journal of Thoracic Disease. 2017. 9: 448-451.

Sakai T, Liu L, Teng X, Mukai-Sakai R, Shimada H, Kaji R, Mitan T, Matsumoto M, Toida K, Ishimura K, Shishido Y, Mak TW, Fukui K. Nucling recruits Apaf-1/pro-caspase-9 complex for the induction of stress-induced apoptosis. Journal of Biological Chemistry. 2004. 279: 41131-41140.

Siegel RL, Miller KD, Jemal A. Cancer statistics, 2016. CA: A Cancer Journal for Clinicians. 2016. 66: 7-30.

Shakeri R, Kheirollahi A, Davoodi J. Apaf-1: Regulation and function in cell death. Biochimie. 2017. 135: 111-1125.

Tiedemann RE, Schmidt J, Keats JJ, Shi CX, Zhu YX, Palmer SE, Mao X, Schimmer AD, Stewart AK. Identification of a poten natural triterpenoid inhibitor of proteosome chymotrypsin-like activity and NF-kappaB with antimyeloma activity in vitro and in vivo. Blood. 2009. 113: 4027-4037. 
Tomicic MT, Christmann M, Fabian K, Kaina B. Apaf-1 deficient mouse fibroblasts are resistant to MNNG and MMS-induced apoptotic death without attenuation of Bcl-2 decline. Toxicology and Applied Pharmacology. 2005. 207: 117-122.

Wang X, Simpson ER, Brown KA. p53: Protection against tumor growth beyond effects on cell cycle and apoptosis. Cancer Research. 2015. 75: 5001-5007.

Yousef BA, Guerram M, Hassan HM, Hamdi AM, Zhang LY, Jiang ZZ. Pristimerin demonstrates anticancer potential in colorectal cancer cells by inducing G1 phase arrest and apoptosis and suppressing various pro-survival signaling proteins. Oncology
Reports. 2016. 35: 1091-1100.

Yousef BA, Hassan HM, Zhang LY, Jiang ZZ. Anticancer potential and molecular targets of pristimerin: A Mini-Review. Current Cancer Drug Targets. 2017. 17: 100-108.

https://doi.org/10.15616/BSL.2018.24.1.15

Cite this article as: Seo HW, Park JH, Lee JY, Park HJ, Kim JK. Pristimerin, a Naturally Occurring Triterpenoid, Exerts Potent Anticancer Effect in Colon Cancer Cells. Biomedical Science Letters. 2018. 24: 15-22. 